\title{
Delving into Vertebrate Serpins for Understanding their Evolution
}

\author{
Abhishek Kumar \\ Cellular Genetics, Faculty of Technology, University of Bielefeld, D-33501, Bielefeld, Germany \\ Email: abhishek.abhishekkumar@gmail.com
}

\begin{abstract}
The superfamily of serine proteinase inhibitors (serpins) is involved in an array of fundamental biological processes such as blood coagulation, cell differentiation, cell migration, complement activation, embryo implantation, fibrinolysis, angiogenesis, and inflammation, and tumor suppression. Vertebrate serpins can be conveniently classified into six subgroups, based on three independent biological features - genomic organization, diagnostic amino acid sites and rare indels. The present vertebrate serpins are derived from an original serpin most probably by intron insertion and we are trying to reconstruct the phylogeny of vertebrate serpin and looking for the reconstruction of original vertebrate gene(s). We started with fish genomes and characterized fish serpins and assigned orthology with respect to human serpins. Most fish serpins are characterised as stereotype vertebrate serpins with some interesting exceptions which suggest that either there are some fish-specific serpins or some fish serpins do not have human orthologs.
\end{abstract}

\section{Introduction}

Serpins stand for serine protease inhibitors and there are around 500 serpins which from -- bacteria, viruses, plants and animals, which constitute a superfamily of proteins having low sequence similarity, but which are structurally conserved and often similar function. Serpins are generally 350-500 amino acids long, consisting of three beta-sheets and eight to nine alpha-helices, but the hallmark of serpins is 20 amino acid long C-terminal motif - reactive centre loop (RCL) where proteases bind and cleave and in turn get inactivated (1-2). Several aspects of the serpins suggest that they might provide an excellent vehicle for studying important questions about protein evolution, structure, and function

- Extensive functional radiation among serpin proteins. Serpins regulate numerous separate intracellular and extracellular processes, including blood coagulation, cell differentiation, cell migration, complement activation, embryo implantation fibrinolysis, angiogenesis, inflammation, tumor suppression and other functions (3). While most serpins appear to act as protease inhibitors (4), some have lost this inhibitory role and function instead in blood pressure regulation (angiotensinogen) or hormone binding (corticosteroid-binding globulin).

- There is considerable gene clustering. For example, the genes coding for mammalian 1antitrypsin like serpins and ov-serpins are clustered and organized into two clusters. The genes in these clusters lie in proximity on the same chromosome, suggesting that they might have arisen by tandem duplications from a common precursor (5-6). However, in spite of their close physical proximity, these proteins have disparate functions, raising interesting questions about the evolution of gene function and expression.

- Serpin genes exhibit a variety of distinct exon-intron patterns. The exon-intron structure of genes may contain important phylogenetic signals, and several authors have suggested the feasibility of evolutionary classifications based on comparative intron positioning (7-8). The serpin genes do exhibit differential exonintron organization (9-12).

- Serpins exhibit dynamic structural properties. Serpins exist in active, cleaved, and latent forms (1). 


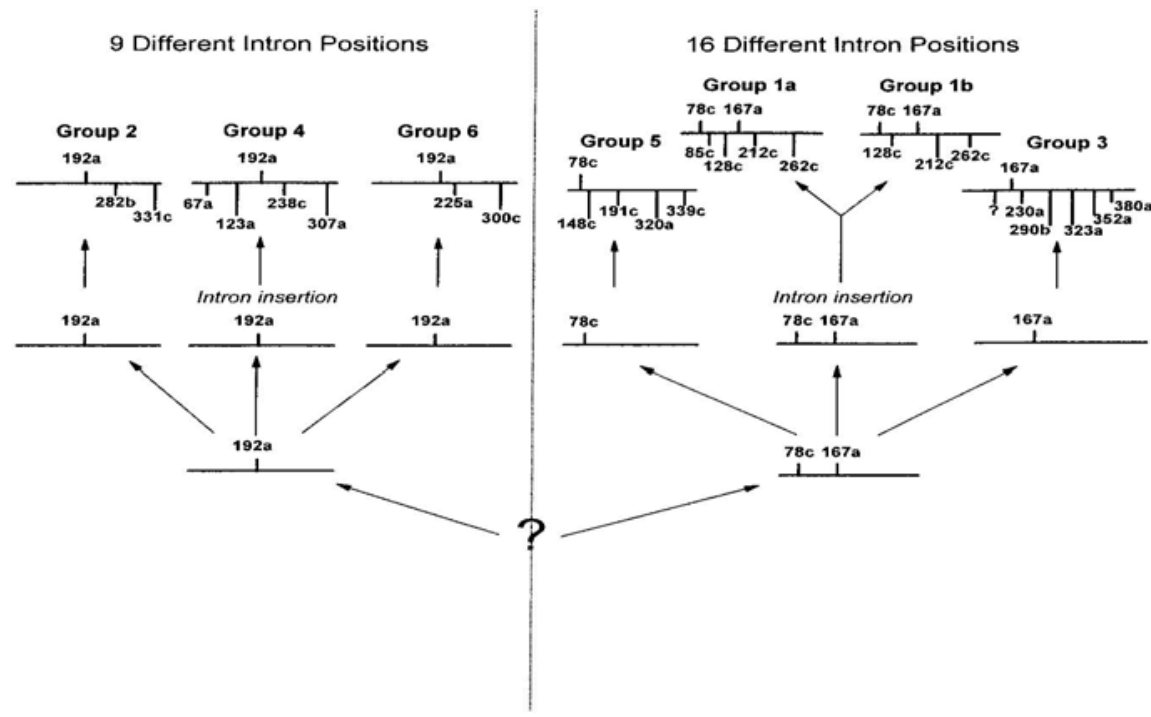

Fig 1. Phylogeny of vertebrate serpins based on gene structure, diagnostic amino acid sites, and indels suggest that present day vertebrate serpins have evolved from a single primordial gene by massive intron insertions (adopted from reference 12).

Thus, these properties suggest that serpins constitute an important group for comparative evolutionary analyses of protein structure and function. However, such studies require a well-documented phylogeny upon which to base speculations about structural and functional variation. The human genome encodes for at least 35 members that evolutionarily segregate into nine (A-I) distinct clades (13). Based on exon-intron organization, family-specific diagnostic amino acid sites, and rare indels, the vertebrate serpin evolution have been deduced (12), which suggested that present day vertebrate serpins can be classified into six groups which have arise from a single primordial gene by massive intron insertions before or during early vertebrate radiation as shown in figure 1.

In our work, we are trying to reconstruct the vertebrate serpin phylogeny and to find the answer to the following questions: (a) How and when did the supposed original vertebrate serpin gene(s) arise, (b) How and when did the six groups separate , (c) What is the mechanism of intron insertion in serpin genes? To answer of these questions, we started working on fish genomes like Fugu, zebrafish, and Tetraodon and we will move towards evolutionary important organisms, whose genomes are available, like chicken and Xenopus. Here, we are representing some of our results of studies on fish serpins, strategies in orthology assignments, and we seek your suggestions and ideas to carry out this project further.

\section{Materials and Methods}

\section{Databases}

We used the following fish genome databases: Fugu genome, Danio genome and Tetraodon genome. For differential comparison and annotation based data, we used NCBI (18), ENSEMBL (19) and SWISSPROT EXPASY (20) databases.

\section{Searching Fish Serpins}

Initially we looked at the genome databases of Fugu, Danio and Tetraodon for annotation of genomic sequences carrying serpin genes, but the quality of annotation was not good enough to have confidence of having all serpins from each fish genome. Therefore, we use the Genlight platform (21) for comparison and finding out all possible serpin genes using BLAST (22). Secondly, we scanned for three typical serpins motifs present in serpin sequences.

\section{Gene Prediction}

Gene structure predictions are mostly made through combination of GENSCAN (23-24), GenomeScan (23-24), and GeneWise (25) using genomic sequences. 
Mapping intron positions

Intron positions of each serpin are mapped with respect to conserved regions of alpha-1-antitrypsin.

\section{Orthology Assignments}

Orthology is assigned with different independent parameters - percentage identity at protein sequence level, group-specific exon-intron structures, presence of gene specific signature motifs, by presence or absence of signal peptide, used group-specific diagnostic sites, reactive centre loop characteristics, Genlight's orthology finding tool, which uses bidirectional BLAST and synteny between human and fish genomes which gives idea of gene order on chromosome as serpins are found in a specific order in mammalian genomes.
For that serpin of interest are aligned with alpha-1antitrypsin using ClustalW (26) and the introns in genomic sequences positioned with help of GeneWise (25) onto this aligned protein sequences.

\section{Results and Discussions}

We found 17 serpins in the Fugu genome which are also present in Tetraodon and zebrafish. Subsequently we assigned orthology to each fish serpin with human serpins. Most of time we found these have orthologs in human genome as listed in table 1 . Fish serpins tend to classify in same fashion into 6 groups of serpins as mammalians, the exon-intron structure is similar as of higher vertebrates found from the same strategies used in reference 12 .

Table 1. List of Fugu serpins, with gene identifiers, scaffold Id where corresponding genes are found and possible orthology with human genes. Bold face indicates that the confidence in assigning orthology is high. Brackets organism name indicate ortholog found in that organism but not human ortholog.

\begin{tabular}{|c|c|c|c|c|c|}
\hline S. No. & $\begin{array}{c}\text { Fugu } \\
\text { Serpents }\end{array}$ & $\begin{array}{l}\text { Gene Id (Fugu v3 } \\
\text { genome database) }\end{array}$ & Scaffold Id & $\begin{array}{c}\text { Clone name } \\
\text { (UK Fugu } \\
\text { Database) }\end{array}$ & Human Ortholog \\
\hline 1 & Fu-Spn-1 & FRUP00000156735 & scaffold_2913 & FM:M002913 & Heat Shock Protein 47 \\
\hline 2 & Fu-Spn-2 & FRUP00000155065 & scaffold_757 & FM:M000757 & Alpha-1-antitrypsin (Xenopus) \\
\hline 3 & Fu-Spn-3 & FRUP00000161527 & scaffold_2007 & FM:M002007 & Neuroserpin precursor \\
\hline 4 & Fu-Spn-4 & FRUP00000132180 & scaffold_188 & FM:M000188 & Z-dependent protease inhibitor \\
\hline 5 & Fu-Spn-5 & FRUP00000137160 & scaffold_417 & FM:M000417 & Glia derived nexin precursor \\
\hline 6 & Fu-Spn-6 & FRUP00000149263 & scaffold_385 & FM:M000385 & Heparin cofactor II \\
\hline 7 & Fu-Spn-7 & FRUP00000160285 & scaffold_6239 & FM:M006239 & Alpha1-Antitrypsin \\
\hline 8 & Fu-Spn-11 & FRUP00000140727 & scaffold_508 & FM:M000508 & Angiotensinogen \\
\hline 9 & Fu-Spn-12 & FRUP00000141273 & scaffold_1026 & FM:M001026 & Pigment epithelium-derived factor \\
\hline 10 & Fu-Spn-14 & FRUP00000162952 & scaffold_154 & FM:M000154 & Alpha-2-antiplasmin precursor \\
\hline 11 & Fu-Spn-17 & FRUP00000155064 & scaffold_757 & FM:M000757 & Antitrypsin \\
\hline 12 & Fu-Spn-18 & FRUP00000146289 & scaffold_641 & FM:M000641 & Antitrypsin \\
\hline 13 & Fu-Spn-35 & FRUP00000131353 & scaffold_110 & FM:M000110 & Human LE1 \\
\hline 14 & Fu-Spn-36 & FRUP00000163136 & scaffold_2405 & FM:M002405 & Human LE1 \\
\hline 15 & Fu-Spn-37 & FRUP00000138778 & scaffold_5139 & FM:M005139 & Human LE1 \\
\hline 16 & Fu-Spn-38 & FRUP00000165249 & scaffold_1226 & FM:M001226 & Antithrombin III \\
\hline 17 & Fu-Spn-39 & FRUP00000133449 & scaffold_3139 & FM:M003139 & C1-Inhibitor \\
\hline
\end{tabular}


(a) Percent sequence indentity $=\mathbf{5 3}$

(b) Signature motifs: Acidic repeats and conserved helix-D.

\section{(c) Exon-intron Structure.}

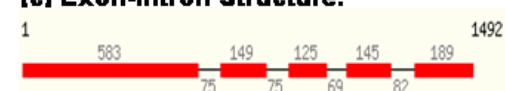

(d) Mapping of intron positions for HCll.
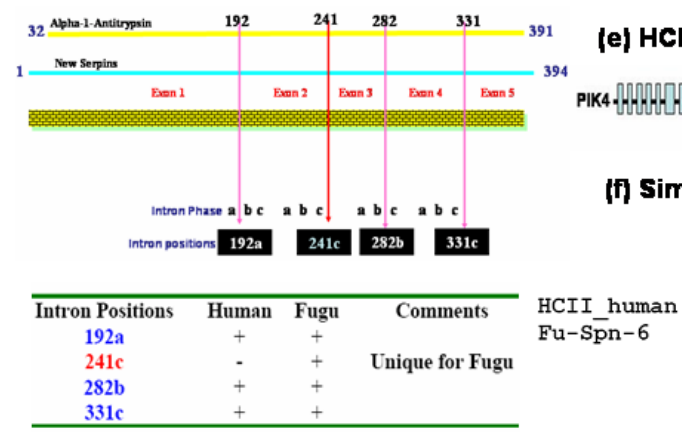

(e) HCll lies in a big intron of PIK4.

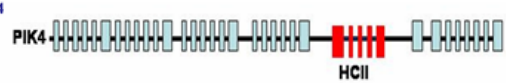

(f) Similar RCL.

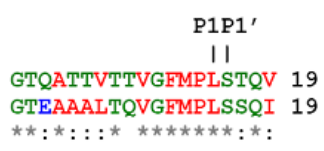

Fig 2. Assigning Orthology for Fu-Spn-6 based of following independent parameters: (a) It has 53\% sequence identity with human heparin cofactor II (HCII). (b) Also carrying two signature motif of heparin cofactor II acidic repeats and conserved helix-D. (c) Exon-intron structure is characteristic for group 2 members. (d) Intron phasing is denominated as a, b and c for first, second and third nucleotide from where introns are spliced out. Table shows the intron positions in Fu-Spn-6 which is similar to human HCII except for 241c. (e) The human HCII gene lies within bigger intron of another gene called PIK4 and this situation is found in fishes as well. (f) Fu-Spn-6 has very similar RCL motif as human HCII. Therefore, FuSpn-6 is indeed the ortholog of human HCII.
Normally we achieved a good confidence level by assigning orthology with help of several independent parameters as shown in figure 2 - the case of Fu-Spn6, which has human heparin cofactor II (HCII) as an ortholog, as indicated by $53 \%$ sequence identity, presence of acidic repeats and conserved helix-D which are functionally very important, exon-intron structure similarities. In human the HCII gene lies in a bigger intron of a gene called phosphatidylinositol 4-kinase (PIK4), same is in case of fishes as well, we used this as marker for HCII orthology. Finally of FuSpn-6 has very similar RCL as of HCII of human. These parameters indeed suggest that human HCII is ortholog of Fu-Spn-6. In some cases we have found human orthologs very easily as we had many parameters about genes. But in some cases especially in group 2 members, it was tough to indicate orthologs. In some cases there is no human ortholog like for Fu-Spn-2 which has alpha-1-antitrypsin like gene from Xenopus as ortholog which has extra Hisrich domain which helps in estrogens binding. We found that fishes have serpins which are classified into 6 groups as in higher vertebrates. There are just 17 serpin genes in fishes, while 35 in human, 59 in mouse and 42 in rat, suggest that serpin genes dont follow whole genome duplication in fishes during early vertebrate evolution (28).
There are there serpin genes in fishes which are orthologous to Leukocyte elastase inhibitor of group 1 , which is highly expanded by tandem gene duplication on the same chromosomes in human and still further expanded in rat and mouse (29). This is a very surprising result that fishes have just one of these ov-serpins while in mammalians there are wide array of ov-serpins which are functionally highly diversified. Group 2 serpins in fishes are again just seven while there are more in mammals that suggest serpins are differentially expanded into different organisms supposedly because of organism based requirements.

\section{Conclusions}

The fish serpins tend to group in a similar fashion as higher vertebrates, since they have same exon-intron structures and conserved intron positions with some interesting exceptions which might be interesting clues to serpins evolution which is under investigation. Assigning orthology to serpin gene is not easy and it requires a big amount of gene specific information. By now we have assigned orthology to fish serpins and analysed these genes and it seems our proposed vertebrate serpins gene is evolved before fish and higher vertebrate separation around $450 \mathrm{My}$ ago ( where fish and mammals separated ) and serpin genes do not follow whole genome duplication in fishes during early vertebrate evolution. 


\section{References}

1. Irving, J. A., Pike, R. N., Lesk, A. M., \& Whisstock, J. C. (2000). Phylogeny of the serpin superfamily: implications of patterns of amino acid conservation for structure and function. Genome Research, 10 (12), 1845-1864.

2. Gettins G.W.P, (2002). Serpin structure, Mechanism, and Function. Chem Review 102. 4751-4803.

3. Potempa, J., Korzus E., and Travis. J. (1994). The serpin superfamily of proteinase inhibitors: structure, function, and regulation. J. Biol. Chem. 269:15957-15960

4. Wilczynska M., M. Fa, P. I. Ohlsson, T. Ny, (1995). The inhibition mechanism of serpins. Evidence that the mobile reactive center loop is cleaved in the native protease-inhibitor complex J. Biol. Chem 270:29652-29655

5. Billingsley G. D., M. A. Walter, G. L. Hammond, D. W. Cox, (1993). Physical mapping of four serpin genes: alpha 1-antitrypsin, alpha 1antichymotrypsin, corticosteroid-binding globulin, and protein C inhibitor, within a 280-kb region on chromosome I4q32.1 Am. J. Hum. Genet 52:343-353

6. Rollini P., R. E. Fournier, (1997). A 370-kb cosmid contig of the serpin gene cluster on human chromosome 14q32. 1: molecular linkage of the genes encoding alpha 1-antichymotrypsin, protein C inhibitor, kallistatin, alpha 1antitrypsin, and corticosteroid-binding globulin. Genomics, 46:409-415

7. Long M., S. J. de Souza, W. Gilbert, (1995). Evolution of exon-intron structure of eukaryotic genes Curr. Opin. Genet. Dev 5:774-778

8. Logsdon J. M. Jr., A. Stolzfus, W. F. Doolittle. (1998) Molecular evolution: recent cases of spliceosomal intron gain. Curr. Biol 8:560-563

9. Bao, J.-J., R. N. Sifers, V. J. Kidd, F. D. Ledley, and S. L. C. Woo. (1987). Molecular evolution of serpins: homologous structure of the human $\boldsymbol{\alpha}_{1}$ antichymotrypsin and $\boldsymbol{\alpha}_{1}$-antitrypsin genes. Biochemistry 26:7755-7759.

10. Ragg H., G. Preibisch, (1988). Structure and expression of the gene coding for the human serpin hLS2. J. Biol. Chem 263:12129-12134

11. Remold-O'Donnell E., (1993).The ovalbumin family of serpin proteins FEBS Lett 315:105-108

12. Ragg H., T. Lokot, P. B. Kamp, Atchley, W.R., Dress A.(2001) Vertebrate serpins: construction of a conflict-free phylogeny by combining exon- intron and diagnostic site analyses Mol. Biol. Evol 18:577-584

13. Silverman, G. A., Bird, P. I., Carrell, R. W., Church, F. C., Coughlin, P. B., Gettins, P. G. W., Irving, J. A., Lomas, D. A., Luke, C. J., Moyer, R. W., Pemberton, P. A., Remold-O’Donnell, E.,Salvesen, G. S., Travis, J., and Whisstock, J. C. (2001). The serpins are an expanding superfamily of structurally similar but functionally diverse proteins. Journal of Biological Chemistry, 276, 33293-33296.

14. Fugu genome v3 database, http://genome.jgipsf.org/fugu6/fugu6.home.html.

15. Fugu genome sequencing project, http://fugu.hgmp.mrc.ac.uk/.

16. Danio rerio genome, http://www.sanger.ac.uk/Projects/D_rerio/.

17. Tetraodon genome, http://www.genoscope.cns.fr/externe/tetranew/dat a/assembly/.

18. National center for biotechnological information, http://www.ncbi.nlm.nih.gov/.

19. Ensemble, http://www.ensembl.org/.

20. Swissprot, http://us.expasy.org/sprot/.

21. Genlight, http://piranha.techfak.uni-bielefeld.de/

22. Altschul S.F., Gish W., Miller W., Myers E.W., Lipman D.J. (1990). Basic local alignment search tool.

J Mol Biol 215: 403-10.

23. Burge, C., and Karlin, S. (1997). Prediction of complete gene structures in human genomic DNA. J. Mol. Biol. 268, 78-94.

24. Burge, C. B., and Karlin, S. (1998). Finding the genes in genomic DNA.Curr. Opin. Struct. Biol. 8, 346-354.

25. Genewise, http://www.ebi.ac.uk/Wise2/\#

26. Higgins D., Thompson J., Gibson T.Thompson J.D., Higgins D.G., Gibson T.J. (1994). CLUSTAL W: improving the sensitivity of progressive multiple sequence alignment through sequence weighting, position-specific gap penalties and weight matrix choice. Nucleic Acids Res. 22, 4673-4680.

27. Yoshida, K., Suzuki, Y. and Sinohara, H. (1999) Cloning and comparative sequence analysis of Xenopus laevis alpha1-antiproteinase. J. Biochem. Mol. Biol. Biophys. 3, 59-63.

28. Ohno, S. (1970). Evolution by gene duplication. Springer-Verlag, NY.

29. Puente XS, Lopez-Otin C. A (2003). Genomic analysis of rat proteases: a comparative genomic approach. Nat Rev Genet. 4, 544-58. 